\title{
Eritema indurado como forma de presentación de un caso de leucemia mielomonocítica crónica
}

\author{
J. M. RAYA SÁNCHEZ, M. J. RODRÍGUEZ SALAZAR, M. L. BRITO BARROSO, \\ F. GUIMERÁ MARTÍN-NEDA ${ }^{1}, \mathrm{H}$. ÁLVAREZ-ARGÜELLES CABRERA², \\ L. HERNÁNDEZ NIETO
}

Servicios de Hematología, ${ }^{1}$ Dermatología $y^{2}$ Anatomía Patológica. Hospital Universitario de Canarias. La Laguna (Tenerife)

\author{
ERYTHEMA INDURATUM AS PRESENTING FORM IN A CASE OF \\ CHRONIC MYELOMONOCYTIC LEUKEMIA
}

\begin{abstract}
RESUMEN
La leucemia mielomonocítica crónica (LMMC) constituye un proceso oncohematológico de naturaleza mixta, mieloproliferativa y mielodisplásica, siendo su forma habitual de presentación consecuencia, generalmente, de las citopenias en sangre periférica (síndrome anémico, infecciones o diátesis hemorrágica). La afectación cutánea en pacientes con síndromes mielodisplásicos o leucemias crónicas, ya bien sea específica o inespecífica, es una circunstancia excepcional, teniendo lugar más frecuentemente en estadios avanzados de la enfermedad como preámbulo a una transformación del proceso crónico en leucemia aguda. El reconocimiento y el diagnóstico precoces del tipo de afectación cutánea en cualquier paciente con síndrome mielodisplásico o mieloproliferativo crónico, obtenido dicho diagnóstico mediante biopsia de la lesión, resulta de gran importancia, pues conlleva un claro significado pronóstico y terapéutico. Describimos a continuación el caso de un paciente que presentó una lesión cutánea inespecífica, un eritema indurado de Bazin, como manifestación inicial de una LMMC; se comentan aspectos diagnósticos, terapéuticos y evolutivos del mismo.
\end{abstract}

PALABRAS CLAVE: Leucemia mielomonocítica crónica. Eritema indurado. Paniculitis lobulillar.

\begin{abstract}
Chronic myelomonocytic leukemia (CMML) is an oncohematologic disease with a mixed nature, myeloproliferative and myelodysplastic, and presenting features are usually the consequence of peripheral blood cytopenias (anemic syndrome, infections or bleeding). Specific or nonspecific cutaneous involvement in patients with myelodysplastic syndromes or chronic leukemias is exceptional, and it takes place often in advanced stages of the disease, as a preample of a transformation from chronic illness to acute leukemia. Recognition and early diagnosis of the skin lesion by cutaneous biopsy, in every patient with myelodysplastic or myeloproliferative disease, have therapeutic and prognostic significance. We describe a patient who presented with a non-especific cutaneous lesion, Bazin's erhytema induratum, as initial manifestation of chronic myelomonocytic leukemia; we also comment diagnostic, therapeutic and clinical evolution aspects.
\end{abstract}

KEY WORDS: Chronic myelomonocytitc leukemia. Erhytema induratum. Lobular panniculitis.

Raya Sánchez, JM, Rodríguez, Salazar MJ, Brito Barroso ML, Guimerá Martín-Neda F, Álvarez-Argüelles Cabrera H, Hernández, Nieto L. Eritema indurado como forma de presentación de un caso de leucemia mielomonocítica crónica. An Med Interna (Madrid) 2004; 21 : 17-19.

\section{INTRODUCCIÓN}

En la clasificación publicada recientemente de tumores de tejidos hematopoyéticos y linfoides (1), parte de la más amplia Clasificación de Tumores de la Organización Mundial de la Salud (OMS), la leucemia mielomonocítica crónica (LMMC) es encuadrada en la categoría de enfermedades mielodisplásicas/mieloproliferativas, reconociéndose en ella un "solapamiento" (ya defendido por algunos autores) entre ambos tipos de procesos (2). Esta enfermedad afecta siempre la médula ósea y la sangre de los pacientes, y los órganos extramedulares más frecuentemente infiltrados son bazo, hígado, piel y ganglios linfáticos. La presentación inicial de LMMC como enfermedad cutánea (ya se trate de lesiones específicas o inespecíficas) es una posibilidad recogida en la literatura en diversos trabajos. La forma de presentación como eritema indurado, sin embargo, es sumamente extraordinaria. Describimos el caso de un paciente que presentó un eritema indurado como primera manifestación, inespecífica, de una LMMC.

\section{CASO APORTADO}

Varón de 64 años, sin antecedentes personales de interés, que es remitido a la consulta de Dermatología por cuadro de unos 45 días de evolución de nódulos cutáneos recidivantes, indoloros e indurados

Trabajo aceptado: 30 de julio de 2003 
localizados en miembros inferiores (Fig. 1). No impresionaba de enfermedad, ni refería pérdida de peso, fiebre, ni síndrome constitucional. En la exploración física destacaban varias lesiones en forma de placas nodulares subcutáneas de 1 a $3 \mathrm{~cm}$. de diámetro, eritematovioláceas, simétricas, duras e indoloras, localizadas en ambas caras internas de muslos, pantorrilla, abdomen y hombro izquierdo. No presentaba adenopatías ni visceromegalias y se apreciaban signos de tromboflebitis superficial en miembro inferior derecho. En las pruebas de laboratorio se objetivó: leucocitos $12,3 \times 10^{9} / \mathrm{L}$ (neutrófilos 6,8, linfocitos 1,1, monocitos 4.3, eosinófilos 0,1), Hb 120 g/L, VCM 109,8 fL; Hto. 32\%; plaquetas 133 x 10\%/L. Se observaban algunos granulocitos de aspecto pelgueroide (núcleo no segmentado o hiposegmentado) y anisocitosis discreta. Reticulocitos: $2,4 \%$ (totales 86,4 x $10^{\circ} / \mathrm{L}$ ), VSG $60 \mathrm{~mm} / \mathrm{h}$; inmunoglobulinas, proteinograma, pruebas de hemostasia, orina y sedimento, estudio del hierro, vitamina B12 y ácido fólico, normales. Se practicó Eco-doppler de miembros inferiores, demostrándose una tromboflebitis superficial de safena interna del miembro inferior derecho con trombosis de dicha vena e importante componente edematoso del tejido graso circundante, sin signos de trombosis venosa profunda. En varios hemogramas posteriores se mantuvo significativamente elevada la cifra de monocitos en sangre.

Se practicó una biopsia de una lesión cutánea que demostró la

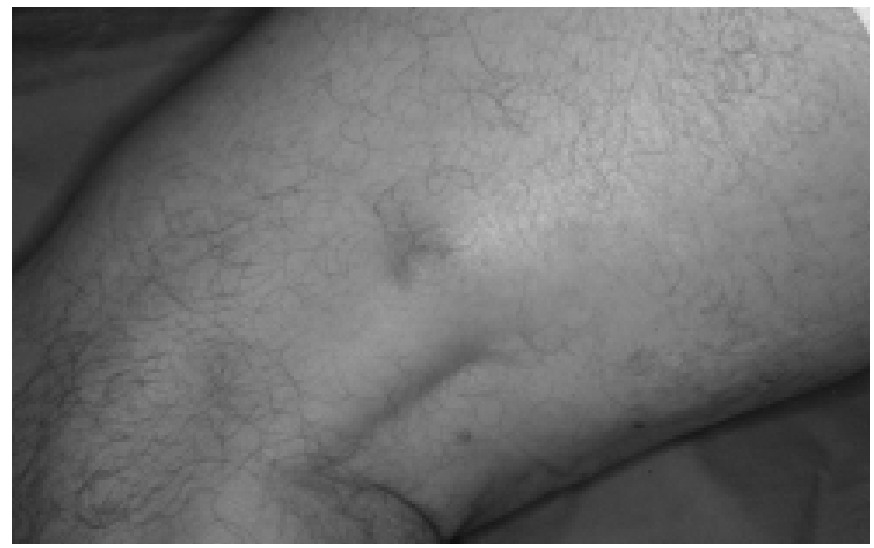

Fig. 1. Nódulo cutáneo localizado en muslo, de $2 \mathrm{~cm}$ de diámetro y color eritemato-violáceo, duro e indoloro a la palpación.

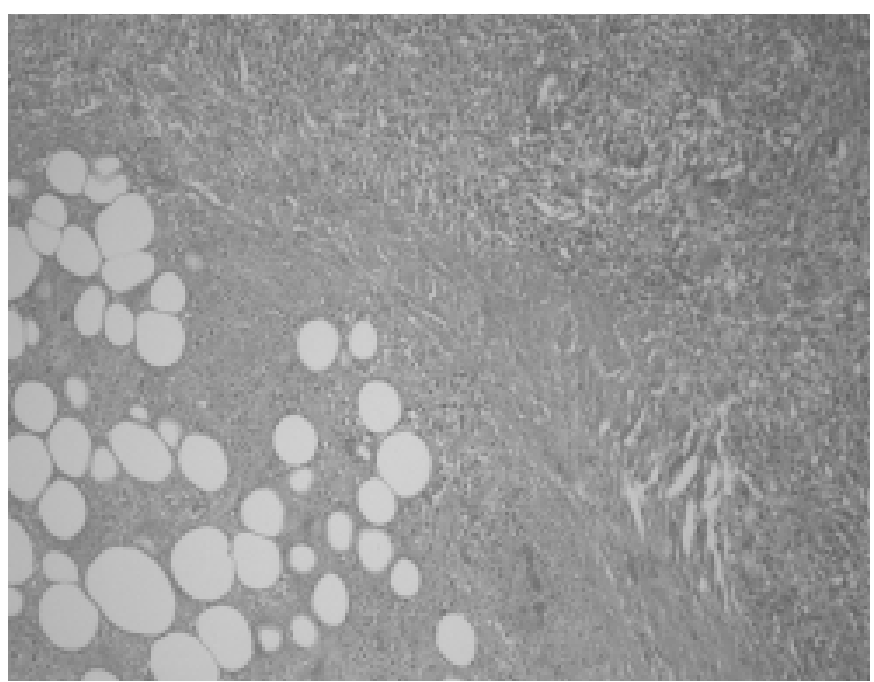

Fig. 2. Necrosis de la grasa dentro del lobulillo graso hipodérmico, y más periféricamente, inflamación mononuclear y algunos granulomas con células gigantes multinucleadas (hematoxilina-eosina, x100). presencia en tejido celular subcutáneo de una paniculitis granulomatosa panlobular con necrosis lobulillar tipo eritema indurado de Bazin o vasculitis nodular (Fig. 2), asociada a trombosis venosa organizada. Se practicó una batería de pruebas diagnósticas para descartar causas Infecciosas (BK, cultivo y análisis por PCR para Mycobacterium tuberculosis: negativos; serologías para infección reciente por Toxoplasma, Legionela, Mycoplasma pneumoniae y para fiebre Q: negativas; ASLO, negativo; Singer Plotz y VDRL, negativos), causas neoplásicas (alfa-feto-proteína, antígeno carcinoembrionario, b2-microglobulina, Ca-19.9, PSA: normales; TAC abdominopélvico: moderado aumento de la densidad de la grasa del mesenterio en flanco derecho que impronta asas intestinales, en probable relación con paniculitis) y enfermedades sistémicas (factor reumatoideo y anticuerpos antinúcleo negativos; niveles normales de $\mathrm{C} 3$ y $\mathrm{C} 4$, proteína $\mathrm{C}$ reactiva, enzima convertidor de angiotensina y alfa-1 antitripsina). La tinción de Ziehl-Neelsen en la muestra de biopsia cutánea (pues el diagnóstico diferencial de la lesión microscópica observada debe establecerse principalmente con una infección cutánea por micobacterias) fue así mismo negativa.

La presencia en sangre de formas granulocíticas displásicas motivó que el paciente fuera remitido a nuestro Servicio, en el que se realizó un aspirado medular, observándose una médula ósea hipercelular con megacariocitos conservados y algún micromegacariocito ocasionalmente, destacando una hiperplasia de la serie monocítica ( $21 \%$ de la celularidad medular global, entre promonocitos y monocitos) e hipoplasia moderada de la serie roja (13\%) con displasia leve (puentes intercitoplasmáticos y algunas formas gigantes), y sobre todo una disgranulopoyesis significativa (citoplasmas francamente agranulares, frecuentes elementos de aspecto pelgueroide, asincronismo madurativo núcleo-citoplasmático y predominio de formas madurativas intermedias); la blastosis constituía sólo un 3\%. El cariotipo fue normal, 46XY. Con todo ello el enfermo se diagnosticó de LMMC con afectación cutánea inespecífica tipo paniculitis lobulillar. El paciente fue tratado con corticoides orales (prednisona 30 $\mathrm{mg} / \mathrm{día}$, con pauta de descenso semanal), resolviéndose las lesiones cutáneas pocos días después de comenzar este tratamiento. En la actualidad, tres años después del diagnóstico y sin tratamiento desde entonces, se encuentra asintomático, con anemia leve, VCM 110.9 $\mathrm{fL}$, plaquetas $143 \times 10^{\%} / \mathrm{L}$, y monocitosis de $2,3 \times 10^{9} / \mathrm{L}$ en sangre, haciéndose controles trimestrales; en médula ósea persiste la displasia trilineal con una blastosis del $6 \%$ y un componente monocítico del $25 \%$.

\section{DISCUSIÓN}

La LMMC, enfermedad oncohematológica caracterizada por la presencia persistente en sangre de una cifra de monocitos superior a $1,0 \times 10^{9} / \mathrm{L}$, ausencia de cromosoma Philadelphia o del gen híbrido BCR/ABL, menos de un $20 \%$ de blastos en médula ósea, y displasia celular que afecte a una o más líneas hematopoyéticas, puede presentarse clínicamente de forma muy heterogénea. La forma habitual de presentación es consecuencia, generalmente, de las citopenias en sangre periférica (síndrome anémico, infecciones asociadas a neutropenia con alteración del funcionalismo del polimorfonuclear, o diátesis hemorrágica asociada a trombopenia) y ocasionalmente se acompaña de hepatomegalia, esplenomegalia o linfadenopatías, derivadas éstas de infiltración leucémica específica. A diferencia de otros síndromes mielodisplásicos (SMD), en los que predominan la leucopenia/neutropenia y los rasgos dishematopoyéticos, la LMMC puede tener como hemos dicho un comportamiento más bien mieloproliferativo, pudiendo existir leucocitosis con desviación a la izquierda, además de la consabida monocitosis.

La afectación cutánea en los pacientes con leucemias cró- 
nicas o SMD constituye una circunstancia rara (3-5), y puede deberse tanto a lesiones inespecíficas (6-8), como más raramente a infiltración propia de la enfermedad $(9,10)$, es decir, como leucemia cutis, siendo en cualquier caso más frecuente en estadios avanzados de la misma. Las lesiones cutáneas suelen aparecer a lo largo de la evolución de la enfermedad, presagiando con frecuencia la transformación a leucemia aguda (11-16), aunque no siempre (17), y más raramente constituyen la manifestación inicial del proceso oncohematológico $(18,19)$. Entre las lesiones cutáneas inespecíficas descritas en asociación a SMD se encuentran las vasculitis (las más frecuentes y polimorfas) de tipo paraneoplásico o asociadas a fármacos, dermatosis neutrofílica, policondritis recidivante, pioderma gangrenoso, síndrome de Sweet, eritema elevatum diutinum, lesiones de hipersensibilidad al frío, pernio, etc.. Recientemente algunos autores han encontrado una asociación estrecha entre LMMC y vasculitis sistémica de vasos de calibre intermedio, anticuerpos citoplasmáticos antineutrófilos (ANCA) negativa, de tipo poliarteritis nodosa $(20,21)$. La presentación clínica no es específica y estas vasculitis sis- témicas deben ser sospechadas cuando un paciente con mielodisplasia desarrolla manifestaciones atípicas. En estos pacientes, las angiografías renales, gastrointestinales o hepáticas, pueden ser de utilidad diagnóstica, y el pronóstico suele ser malo.

Hasta donde nuestro conocimiento alcanza, éste es el segundo caso publicado de paniculitis como forma de presentación de un caso de LMMC (18). La paniculitis del omento sospechada por la imagen del TAC abdominopélvico en nuestro paciente, constituye así mismo una circunstancia excepcional de la que sólo hemos encontrado otra referencia en la literatura (22). El reconocimiento y el diagnóstico precoces del tipo de afectación cutánea en cualquier paciente con SMD, obtenido éste mediante biopsia de la lesión, resulta de gran importancia, pues conlleva un claro significado pronóstico y terapéutico. En algún caso, además, puede tratarse de la forma inicial de manifestación de la enfermedad oncohematológica. Por último, la mayoría de los pacientes con este tipo de dermopatía, eritema indurado, responden bien al tratamiento con corticoides, como así ocurrió en el caso aportado.

\section{Bibliografía}

1. Vardiman JW, Pierre M, Bain B, Bennett JM, Imbert M, Brunning RD, et al. Chronic myelomonocytic leukemia. En: Jaffe ES, Harris NL, Stein H, Vardiman JW, eds. Tumours of haematopoietic and lymphoid tissues. World Health Organization Classification of Tumours. Lyon, IARC Press; 2001, p. 49-52.

2. Germing U, Gattermann N, Minning H, Heyll A, Aul C. Problems in the classification of CMML - dysplastic versus proliferative type. Leuk Res 1998; 22: 871-878.

3. Quaglino D, Di Leonardo G, Furia N, Paglione M, Pasqualoni E. Cutaneous involvement in leukaemic patients. A review of the literature and personal experience. Recenti Prog Med 1997; 88: 415-421.

4. Avivi I, Rosenbaum H, Levy Y, Rowe J. Myelodysplastic syndrome and associated skin lesions: a review of the literature. Leuk Res 1999; 23: 323-330

5. Morand JJ, Lightburn E, Richard MA, Hesse-Bonerandi S, Carsuzaa F, Grob JJ. Skin manifestations associated with myelodysplastic syndromes. Rev Med Interne 2001; 22: 845-853.

6. Kelly JW, Dowling JP. Pernio. A possible association with chronic myelomonocytic leukemia. Arch dermatol 1985; 121: 1048-1052.

7. Dreno B, Gandon P, Bureau B, Milpied N, Barriere H. Skin lesions from hypersensitivity to cold during chronic myelomonocytic leukemia. Br J Dermatol 1986; 115: 607-609.

8. Tural C, Cuxart A, Ribera JM, Millá F. Leucemia mielomonocítica crónica y vasculitis por hipersensibilidad. Med Clin (Barc) 1991; 97: 357.

9. Horiuchi Y, Masuzawa M, Nozaki O, Shibahara N, Shiga T, Yoshida M. Unusual cutaneous lesions associated with chronic myelomonocytic leukaemia. Clin Exp Dermatol 1992; 17: 121-124.

10. Braga D, Manganoni AM, Boccaletti V, Pancera C, Marocolo D, Facchetti F, et al. Specific skin infiltration as first sign of chronic myelomonocytic leukemia with an unusual phenotype. Am Acad Dermatol 1996; 35: 804-807.

11. Duguid JK, Mackie MJ, McVerry BA. Skin infiltration associated with chronic myelomonocytic leukaemia. Br J Haematol 1983; 53: 257-264.

12. O'Connell DM, Fagan WA, Skinner SM, Pulitzer DR, Devillez RL.
Cutaneous involvement in chronic myelomonocytic leukemia. Int $\mathrm{J}$ Dermatol 1994; 33: 628-631.

13. Elenitoba-Johnson K, Hodges GF, King TC, Wu CD, Medeiros LJ. Extramedullary myeloid cell tumors arising in the setting of chronic myelomonocytic leukemia. A report of two cases. Arch Pathol Lab Med 1996; 120: 62-67.

14. Suzuki H, Takahashi T, Hayashi T, Kanamoto H, Hamamoto F, Adachi $\mathrm{M}$, et al. Rapidly progressed chronic myelomonocytic leukemia associated qwith severe skin infiltration. Rinsho Ketsueki 1997; 38: 752-756.

15. Pont V, Miquel FJ, Grau TC, Hernández F, Sánchez-Carazo JL, Aliaga A. Skin involvement in chronic myelomonocytic leukaemia as a predictor of transformation into acute myeloid leukaemia. J Eur Acad Dermatol Venereol 2001; 15: 260-262.

16. Paydas S. Cutaneous lesions and chronic myelomonocytic leukemia (CML) in progression. Ann Hematol 2002; 81: 178

17. Copplestone JA, Oscier DG, Mufti GJ, Hamblin. Monocytic skin infiltration in chronic myelomonocytic leukaemia. Clin Lab Haematol 1986; 8: 115-119.

18. Sanada I, Naito M, Nishimura H, Tomino S, Yamaguchi K, Fujiwara H, et al. Chronic myelomonocytic leukemia with an initial symptom of panniculitis. Rinsho Ketsueki 1985; 26: 2004-2008.

19. Las Heras G, Ribera JM, Millá F, Ferrandiz C, Hernández M. Infiltración cutánea diseminada como primera manifestación de leucemia mielomonocítica crónica. Sangre (Barc) 1990; 35: 142-143.

20. Rosen AM, Haines K 3rd, Tallman MS, Hakimian D, Ramsey-Goldman $\mathrm{R}$. Rapidly progressive cutaneous vasculitis in a patient with chronic myelomonocytic leukemia. Am J Hematol 1995; 50: 310-312.

21. Hamidou MA, Boumalassa A, Larroche C, El Kouri D, Bletry O, Grolleau JY. Systemic medium-sized vessel vasculitis associated with chronic myelomonocytic leukaemia. Semin Arthritis Rheum 2001; 31: 119-126.

22. Disdier P, Harle JR, Herbault GF, Chrestian MA, Horschowski N, Mongin $\mathrm{M}$, et al. Panniculitis of the omentum. Uncommon complication of chronic myelomonocytic leukemia. Ann Med Interne (Paris) 1990; 141: 283-285. 